\title{
Reflecting on light sheets
}

Reflective coverslips can improve spatiotemporal resolution and collection efficiency in diSPIM light-sheet fluorescence microscopy.

In light-sheet fluorescence microscopy (LSFM), a thin layer of a sample is illuminated perpendicularly to the observation objective. This reduces photodamage and out-of-focus fluorescence while providing diffraction-limited resolution. These features, in combination with its high speed, make LSFM an attractive method for time-lapse imaging in a wide range of samples-from cells to living animals and thick tissue samples.

In the popular diSPIM LSFM configuration, two objectives are placed at $45^{\circ}$ angles above the sample. One objective generates the light sheet, while the second objective is used for imaging. This process is repeated with reversed objectives to generate a $3 \mathrm{D}$ image with similar resolution in all directions. Often, high numerical aperture (NA) objectives are used to achieve a high resolution. But these short-working-distance objectives are bulky and can

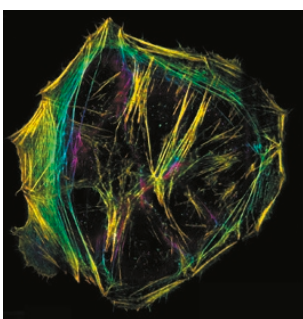

Actin fibers in fixed U20S cells acquired in dual-view geometry on a reflective coverslip. Image adapted with permission from Wu et al. (Springer Nature). be difficult or impossible to arrange in the small available space around the sample, which is a restriction especially when imaging small samples in LSFM.

Hari Shroff and colleagues from the NIH National Institute of Biomedical Imaging and Bioengineering in Bethesda, Maryland, have come up with a simple trick to address this issue-they utilized additional views of the specimen without the need for further expensive objectives or complicating the microscope design. "If one just grew the sample on a mirrored coverslip, [one] would very naturally get these extra views of the sample," says Shroff. The light sheet would reflect off the mirrored coverslip and create a second orthogonal light sheet. This

\section{SEQUENCING}

\section{SEQUENCING DNA, NO MISTAKE}

\section{Building redundancy into fluorogenic sequencing makes for error-free DNA sequence reads.}

Sequencing methods are dominated by commercialized technologies-so much so that most academic researchers consider the field too risky to wade into. That has not deterred Yanyi Huang, a researcher at Peking University, from exploring better ways to generate very accurate DNA reads at high throughput. His forays began after a conversation with his departmental colleague X. Sunney Xie, who published a method in 2011 that couples pyrosequencing with fluorogenic output.

In fluorogenic pyrosequencing, a polymerase is used to synthesize DNA opposite a template strand. A, C, G and T nucleotides are added one at a time with washes in between, and they include chemical modifications that release a fluorescent signal only when a nucleotide is incorporated by the polymerase. The process is rapid, but stretches of the same base are hard to decipher because the signal they generate is not proportional to sequence length.

Huang noticed a key inefficiency in pyrosequencing: "At every given cycle, you have a big probability that the nucleotide you add wouldn't be added, so you have a dark cyclezero signal." He had been reviewing the seminal 1977 paper by Allan Maxam and Walter Gilbert on DNA sequencing by chemical degradation for a class that he taught, and he realized that the authors used signals from overlapping sets of cleaved bases to determine the original sequence. This made him wonder whether nucleotide combinations could be worked into pyrosequencing. "I talked to my student and said, why don't we try to do two bases?" recalls Huang. The idea evolved into sequencing the same template three times with different alternating pairs of nucleotides (e.g., round 1 with $\mathrm{A}+\mathrm{C}$ and $\mathrm{G}+\mathrm{T}$, round 2 with $A+G$ and $C+T$, and round 3 with $A+T$ and $C+G$ ) and removing synthesized strands between rounds. The process generates three orthogonal degenerate sequences, from which the underlying sequence can be decoded unambiguously. 
doubles the acquisition speed, as it allows simultaneous rather than sequential diSPIM imaging. In addition, the fluorescence that would normally be lost through the coverslip would also be reflected, leading to two additional images (one for the original and one for the reflected light sheet). By fusing these images, signal-to-noise ratio and resolution are considerably improved compared to conventional LSFM.

However, the fusion of the four views requires sophisticated modeling and computational postprocessing. "We constructed a very simple model which turned out to be not enough," explains Shroff. At that point, his group teamed up with Patrick La Riviere's computational group at the University of Chicago. La Riviere's group developed an algorithm that modeled the imaging process better than the initial approach. "And then we [went] back and forth [to see] what kinds of features of the model were important for capturing the data," explains Shroff. The model was especially important to remove background fluorescence and account for the fact that the light sheets spread diffractively. The latter also affects nonmirrored LSFM images but has to date been largely ignored, as mathematical tools to model the spreading of the light sheet and correct for it have not been available, according to Shroff. The new deconvolution algorithm can now be used for this purpose. The only price one has to pay is additional computational time. But the researchers have already tuned the algorithm and substantially reduced the postprocessing time since the publication of the paper. And Shroff expects further improvements in this area with the use of faster graphic processing units.

Shroff and colleagues demonstrate the benefits of their approach in a variety of examples, one of them being a freely moving Caenorhabditis elegans embryo. They employed a strain that expresses GCaMP3, thus enabling functional imaging of $\mathrm{Ca}^{2+}$ transients in cells. Extending earlier work from Shroff's group, the researchers were able to visualize $\mathrm{Ca}^{2+}$ transients even in neurites while maintaining a scanning speed of more than $1 \mathrm{~Hz}$.

\section{Christian Schnell}

RESEARCH PAPERS

Wu, Y. et al. Reflective imaging improves spatiotemporal resolution and collection efficiency in light sheet microscopy. Nat. Commun. 8, 1452 (2017).

Sequencing with only two orthogonal sets of nucleotides would be enough to decode sequence, but adding a third round enables error correction. Despite the redundancy, the sequencing reaction does not take longer than traditional pyrosequencing. "You actually use the same amount of reaction compared with single nucleotide addition...but you provide 50\% more information," says Huang.

To implement their method effectively, Huang and his team tested dozens of fluorophores until they found Tokyo Green, a fluorophore with a very high signalto-noise ratio. They also replaced arrayed microchambers in Xie's original work with sequence amplification directly off of a glass flowcell for greater efficiency. The changes reduced the raw sequencing error rate to $\sim 0.5 \%$ in the first 200 nucleotides.

The researchers then developed algorithms using information theory to both detect and correct sequencing errors, something that is not possible with other platforms. They used dynamic programming to efficiently determine a globally optimal decoded sequence from among various possibilities in the presence of error. Their strategy, error correction code (ECC) sequencing, is essentially error free for the first 200 nucleotides. They also found a way to remove error in single-base stretches shorter than eight nucleotides to provide the high raw accuracy needed for ECC sequencing.

The published work uses highly pure identical viral template. But the researchers have since modified their protocol to enable cluster amplification, so that different sequences can be read out in the same flowcell. They are improving other aspects of the technology such as throughput and have licensed it to a company called Cygnus Biosciences. ECC sequencing could ultimately be broadly used to help applications for rare alleles, in which distinguishing true variants from sequencing artifacts is critical.

\section{Tal Nawy}

